\begin{tabular}{|c|c|c|c|}
\hline \multirow{3}{*}{$\begin{array}{r}\text { Case Reports in } \\
\text { Gastroenterology }\end{array}$} & \multirow{2}{*}{\multicolumn{2}{|c|}{ Case Rep Gastroenterol 2017;11:36-41 }} & \multirow[b]{3}{*}{$\begin{array}{l}\text { Karger } \\
\text { Open access }\end{array}$} \\
\hline & & & \\
\hline & $\begin{array}{l}\text { DOI: 10.1159/000455184 } \\
\text { Publisnea onime. January 27, } 2017\end{array}$ & $\begin{array}{l}\text { (c) } 2017 \text { The Author(s) } \\
\text { Published by S. Karger AG, Basel } \\
\text { www.karger.com/crg }\end{array}$ & \\
\hline & $\begin{array}{l}\text { This article is licensed under the } \\
\text { International License (CC BY-NC) } \\
\text { Usage and distribution for commerc }\end{array}$ & $\begin{array}{l}\text { mons Attribution-NonCommercial } 4.0 \\
\text { rger.com/Services/OpenAccessLicense). } \\
\text { quires written permission. }\end{array}$ & \\
\hline
\end{tabular}

\title{
Massive Hemorrhage from Ectopic Duodenal Varices: Importance of a Multidisciplinary Approach
}

\author{
Tyler House Patrick Webb Chad Baarson \\ Naval Medical Center Portsmouth, Portsmouth, VA, USA
}

\section{Keywords}

Ectopic varices · Variceal embolization · Upper gastrointestinal bleeding

\begin{abstract}
Duodenal variceal bleeding is an uncommon complication of portal hypertension that can easily go unrecognized and reach mortality rates as high as $40 \%$. Cirrhosis is the most common cause of duodenal varices. In most cases, duodenal varices occur concomitantly with esophageal varices, further complicating identification with initial endoscopy. Although many modalities have been explored with respect to management and treatment approaches, guidelines have yet to be established owing to the infrequency in which bleeding occurs from ectopic duodenal varices. We present a case of massive duodenal variceal hemorrhage that highlights the complexity of initial diagnosis and ultimately required a transesophageal intrahepatic portosystemic shunt with coil embolization for control of bleeding.

(C) 2017 The Author(s)

Published by S. Karger AG, Basel
\end{abstract}

\section{Introduction}

Cirrhosis is an irreversible process of liver fibrosis and is responsible for significant morbidity and mortality worldwide. As fibrosis progresses, increased resistance to portal venous blood flow develops and portal hypertension (PHT) can occur. Complications of PHT are many, but the most feared and acute is a variceal hemorrhage. The majority of variceal 
House et al.: Massive Hemorrhage from Ectopic Duodenal Varices: Importance of a Multidisciplinary Approach

bleeding occurs in the esophagus or the stomach at a rate of $5-15 \%$ annually [1]. Rarely, varices are found outside of the gastroesophageal region, and these are referred to as ectopic varices (ECV). Varices are found in the duodenum, jejunum, ileum, colon, peristomal, rectum, and many other locations. Duodenal varices are the most common small bowel varices, and cirrhosis is the most common cause. According to one study done by Saad et al. [2] 40\% of patients with PHT undergoing angiography were identified to have duodenal varices. Hemorrhage from ectopic duodenal varices can be difficult to diagnose, often requiring repeat endoscopies and imaging studies. With respect to treatment, there are several modalities utilizing endoscopic techniques that include endoscopic band ligation, sclerotherapy and clipping. Higher hepatic venous pressure gradients are more predictive of treatment failure, at which point interventional radiologic procedures may be required for control of bleeding. Surgery is considered if endoscopic and radiologic techniques have failed. Prognoses vary by site, and much of what it is reported in the literature is based on case reports and series. We present a case of massive duodenal variceal hemorrhage that ultimately required a transesophageal intrahepatic portosystemic shunt (TIPS) with coil embolization for control of bleeding.

\section{Case Report}

A 56-year-old male with decompensated alcoholic cirrhosis Model for End-stage Liver Disease (MELD) 12 at presentation, was transferred to our hospital for management of ongoing hematochezia despite receiving esophageal variceal ligation (EVL) with 3 bands placed for high-risk stigmata varices. The patient had a normal colonoscopy. The presenting hemoglobin $(\mathrm{Hgb})$ from the transferring facility was $6.4 \mathrm{~g} / \mathrm{dL}$ with a rise to $6.7 \mathrm{~g} / \mathrm{dL}$ after 2 units of packed red blood cells. On arrival, repeat esophagogastroduodenoscopy (EGD) revealed dislodgment of the 3 esophageal bands with underlying ulcerations and platelet plugs as well as columns of large esophageal varices. Additionally, there was fresh blood seen in the stomach. Five bands were redeployed in the distal esophagus with no evidence of active bleeding at completion. The patient was transfused to $\mathrm{Hgb} 7.3 \mathrm{~g} / \mathrm{dL}$ but had repeat hematochezia and subsequent decline in $\mathrm{Hgb}$ to $6.2 \mathrm{~g} / \mathrm{dL}$ the following morning. Interventional radiology was consulted for TIPS placement secondary to concerns for failed EVL with rebleeding. Repeat emergent EGD revealed 3 of the newly placed bands were dislodged with no active bleeding. Two medium varices were found in the lower third of the esophagus that were banded and successfully deflated. No bleeding was visualized in the gastric cardia or fundus. Patchy friable mucosa was noted in the proximal duodenum. The patient continued to require blood transfusions and pressor support. A contrast-enhanced CT scan was performed for TIPS surgical planning, as no prior cross-sectional imaging was available. The CT scan revealed a markedly cirrhotic liver, ascites, and previously undiagnosed multiple prominent varices involving the 3rd portion of the duodenum (Fig. 1). The patient was transferred from the CT scanner to the Interventional Radiology Suite and an emergent TIPS was successfully performed. During the TIPS procedure, portal venography demonstrated a mildly prominent coronary vein without obvious esophageal varices. There was significant hepatofugal flow in the portal vein. Additionally, there were prominent duodenal varices noted extending from the proximal superior mesenteric vein with shunting of flow towards the left renal vein (not shown in the figures) which corresponded to the findings seen on CT, and likely the source of the patient's continued bleeding as opposed to the esophageal varices seen on endoscopy (Fig. 2a). Once the TIPS shunt was placed, there was significant reduction 
House et al.: Massive Hemorrhage from Ectopic Duodenal Varices: Importance of a Multidisciplinary Approach

in the portosystemic gradient from 25-30 to 3-5 mm Hg with a noticeable decrease in coronary vein dilation and return of hepatopetal flow of portal blood towards the liver. Given the marked portal system decompression, further interventions were deferred.

On postoperative day 1 , status-post TIPS placement, the patient stabilized but had recurrent hematochezia with an Hgb nadir of $5.0 \mathrm{~g} / \mathrm{dL}$ requiring factor VII for reversal of ongoing coagulopathy and correction of INR from 2.5 to 0.9 . A push enteroscopy was performed. The prior bands were intact, there was no evidence of bleeding or high-risk stigmata, the gastric fundus and cardia were again visualized, and again there was no evidence of varices, bleeding, or high-risk stigmata. Given the findings on CT and on angiography, the third portion of the duodenum was evaluated demonstrating a large varix (Fig. 3) with high-risk stigmata of bleeding seen as evidenced by depressed punctate white spot within an area of superficial erythema.

Owing to the size of the varix and the recurrent hematochezia, endoscopic therapy was deferred, and the patient underwent successful endovascular coil embolization of the duodenal varices (Fig. 2b). There was no further bleeding postoperatively. He developed mild grade 1 hepatic encephalopathy that responded to rifaximin and his MELD score peaked at 23. The patient was discharged home in a stable condition on postoperative day 6 statuspost TIPS. He ultimately required 27 units of packed red blood cell transfusions. The patient was seen most recently at his 3-month postoperative visit with a significant improvement in the MELD score, now at 15. He has been off his rifaximin for a month without any episodes of encephalopathy, does not have ascites, and has returned to work and is fully functional.

\section{Discussion}

ECV are complex pressurized portosystemic venous collaterals occurring within the abdomen excluding the gastroesophageal region and account for up to $5 \%$ of all variceal bleeding. Hemorrhage of these varices can be massive with a mortality rate as high as $40 \%[2,3]$. Ectopic duodenal varices such as seen in our case, account for $17 \%$ of ECV and $25-33 \%$ of ectopic bleeds. Cirrhosis is the most common cause of duodenal varices, accounting for nearly $30 \%$ of cases [2]. It is also thought that duodenal varices may develop after EVL as a result of spontaneous portosystemic shunting to alternate sites. The majority of varices are found in the duodenal bulb and the descending portion of the duodenum [3]. The frequency of bleeding and occurrence decrease in the more distal portions of the duodenum because duodenal varices are located deep in the serosal layer whereas esophageal varices are located in the submucosal layer. ECV can present with hematemesis, hematochezia and as obscure bleeds depending on the location of the varices. Diagnosis can be challenging, and up to $69 \%$ of people with duodenal varices may have coexisting esophageal varices. As many as 5 repeated EGDs have been needed to make the diagnosis [4].

Optimal management is not well established, and there are no treatment guidelines for ECV. Ectopic variceal hemorrhages should be managed in the same way as gastroesophageal hemorrhages with respect to fluid resuscitation, antibiotics, and octreotide with vasopressors if needed [1]. Endoscopic sclerotherapy has been successfully used in the past with good outcomes, although this carries a risk of perforation. EVL has also proved successful in stopping bleeding; however, this approach is limited in varices larger than $15 \mathrm{~mm}$, as was the case with this patient, because obtaining a good view is oftentimes difficult, and isolating the feeding vessels can be problematic. Endoscopic hemoclips can be deployed and control bleeding by providing direct mechanical pressure if the afferent and efferent vessels can be 
isolated. Complications include potentiating bleeding and possible perforation if placed incorrectly. TIPS is effective in controlling bleeding in the acute setting but has no mortality benefit over endoscopic therapies. A small study has demonstrated that patients with hepatic venous pressure gradients greater than $20 \mathrm{~mm} \mathrm{Hg}$ have a significant improvement in survival when performed within $24 \mathrm{~h}$ of an acute variceal hemorrhage but further studies to validate this are needed [1]. TIPS alone appears to be very successful as salvage therapy and decreases the need for repeated procedures, and is proven effective in preventing recurrent esophageal variceal rebleeding [4]. TIPS is also recommended by the American Association for the Study of Liver Diseases for the prevention of rebleeding in ECV. There is however a trend towards increased rebleeding-related mortality seen in the TIPS alone group. When combined with embolization, there is superior outcome over the TIPS alone group in prevention of rebleeding in ECV [2, 5, 6]. Unfortunately, post-TIPS hepatic encephalopathy and shunt stenosis are known complications, and outcomes are worse in patients with more advanced Child-Pugh scores [4].

In conclusion, early angiographic identification and intervention cannot be overemphasized when the primary bleeding site is not identified on initial EGD or when there is a high enough suspicion for ectopic duodenal varices. Angiographic intervention is often indicated in cases of recurrent ectopic variceal bleeding or when persistent ECV remain despite portosystemic decompression as evidenced by a portosystemic pressure gradient decrease to less than $12 \mathrm{~mm} \mathrm{Hg}$ or $25-50 \%$ reduction from baseline measurement [4]. This case highlights the complexity of diagnosing ectopic duodenal varices. An emphasis should be placed on maintaining a high level of suspicion for diagnosis of ectopic variceal bleeds in patients with PHT and ongoing bleeding with early emphasis on angiographic evaluation and intervention.

\section{Statement of Ethics}

There are no ethical conflicts to declare. Informed consent has also been obtained from the involved patient, and there is no identifying patient information in the article.

\section{Disclosure Statement}

The authors certify that they have NO affiliations with or involvement in any organization or entity with any financial interest (such as honoraria; educational grants; participation in speakers' bureaus; membership, employment, consultancies, stock ownership, or other equity interest; and expert testimony or patent-licensing arrangements), or nonfinancial interest (such as personal or professional relationships, affiliations, knowledge or beliefs) in the subject matter or materials discussed in this article.

\section{References}

1 Akhter N, Haskal Z: Diagnosis and management of ectopic varices. Gastrointest Interv 2012;1:3-10.

-2 Saad W, Lipper A, Saad N, et al: Ectopic varices: anatomical classification, hemodynamic classification, and hemodynamic-based management. Tech Vasc Interv 2013;16:108-125.

-3 Almadi M, et al: Ectopic varices. Gastrointest Endosc 2011;74:380-388.

4 Park S, et al: Successful treatment of duodenal variceal bleeding by endoscopic clipping. Clin Endosc 2013;46:403-406. 


\section{Case Reports in Gastroenterology}

\begin{tabular}{l|l}
\hline Case Rep Gastroenterol 2017:11:36-41 \\
\hline DOI: 10.1159/000455184 & $\begin{array}{l}\text { @ 2017 The Author(s). Published by S. Karger AG, Basel } \\
\text { www.karger.com/crg }\end{array}$ \\
\hline
\end{tabular}

House et al.: Massive Hemorrhage from Ectopic Duodenal Varices: Importance of a Multidisciplinary Approach

5 Gaba R, et al: Rebleeding rate following TIPS for variceal hemorrhage in the Viatorr era: TIPS alone versus TIPS with variceal embolization. Hepatol Int 2010;4:749-756.

6 Vangeli M, et al: Bleeding ectopic varices - treatment with transjugular intrahepatic porto-systemic shunt (TIPS) and embolisation. J Hepatol 2004;41:560-566.
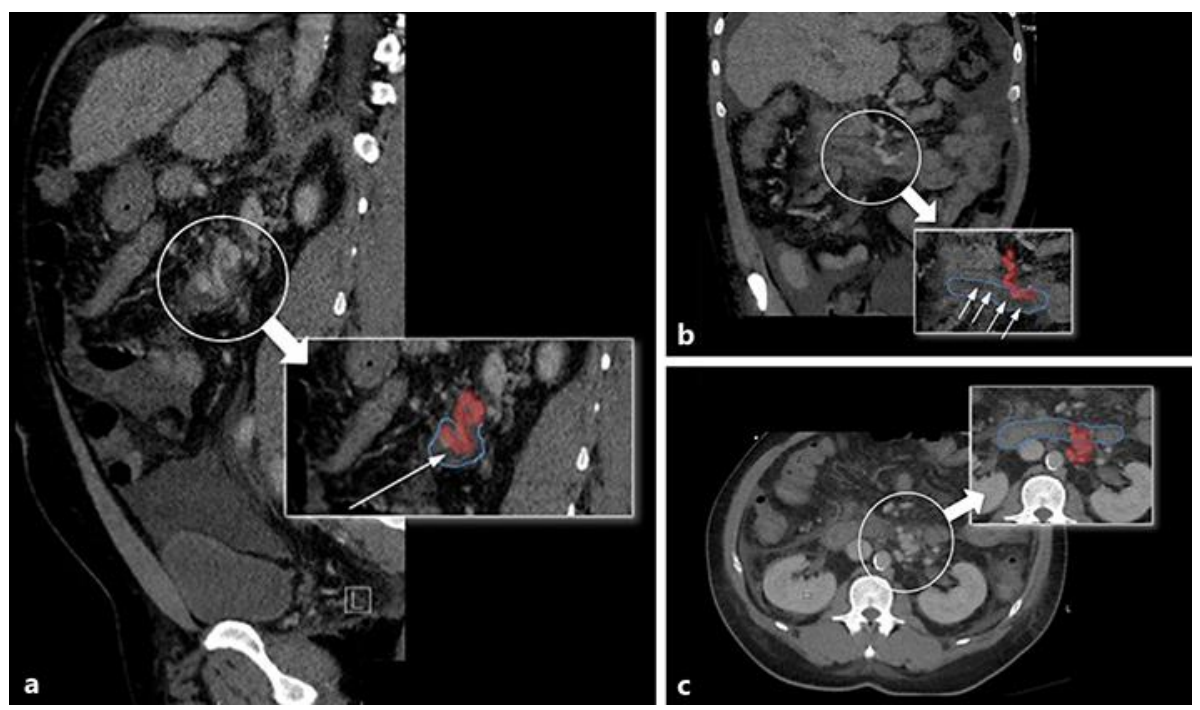

Fig. 1. The following images are from a contrast-enhanced CT of the abdomen demonstrating the tortuous varix (red) protruding into the 3rd portion of the duodenum (blue outlining the duodenal border) with white arrow demonstrating lumen of the duodenum. a Sagittal image. b Coronal image at the region of varices. c Two axial CT slices at the level of the third portion of the duodenum. 

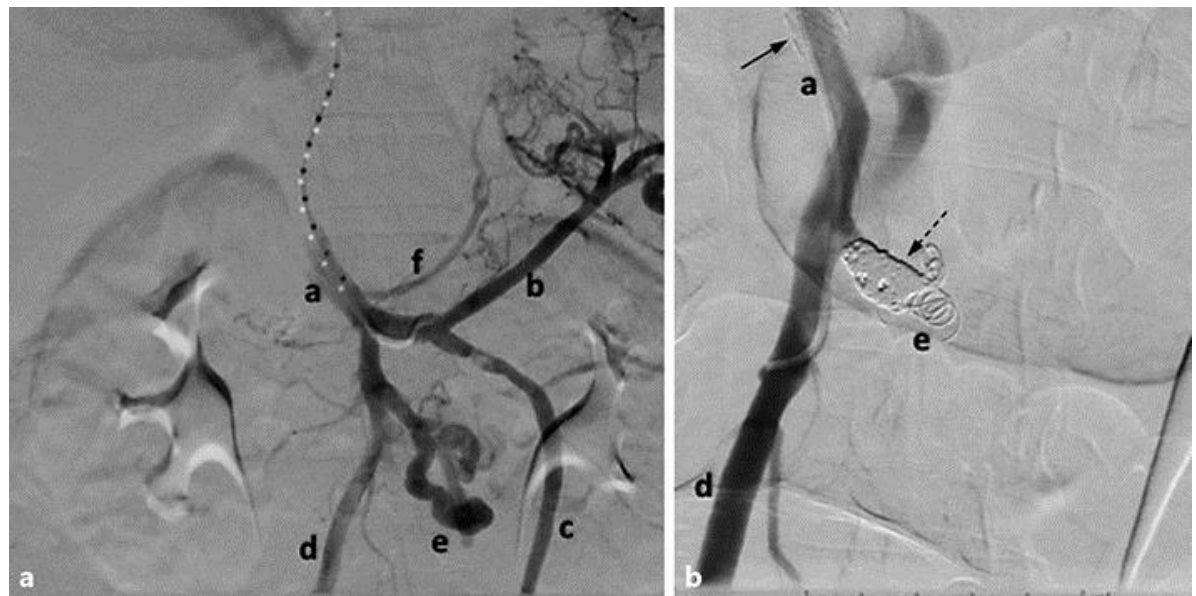

Fig. 2. a Digital subtraction angiographic image performed during the transjugular intrahepatic portosystemic shunt (TIPS) procedure demonstrates prominent duodenal varices extending from the inferior mesenteric vein (IMV). Additionally, there is a small coronary vein noted from the main portal vein (MPV). b Focused digital subtraction image of the MPV and IMV confluence after TIPS placement (solid arrow) as well as postembolization of the duodenal varices (dashed arrow) demonstrating no further flow within the varices. a, main portal vein; $b$, splenic vein; c, superior mesenteric vein; $d$, inferior mesenteric vein; e, duodenal varices; f, coronary vein.
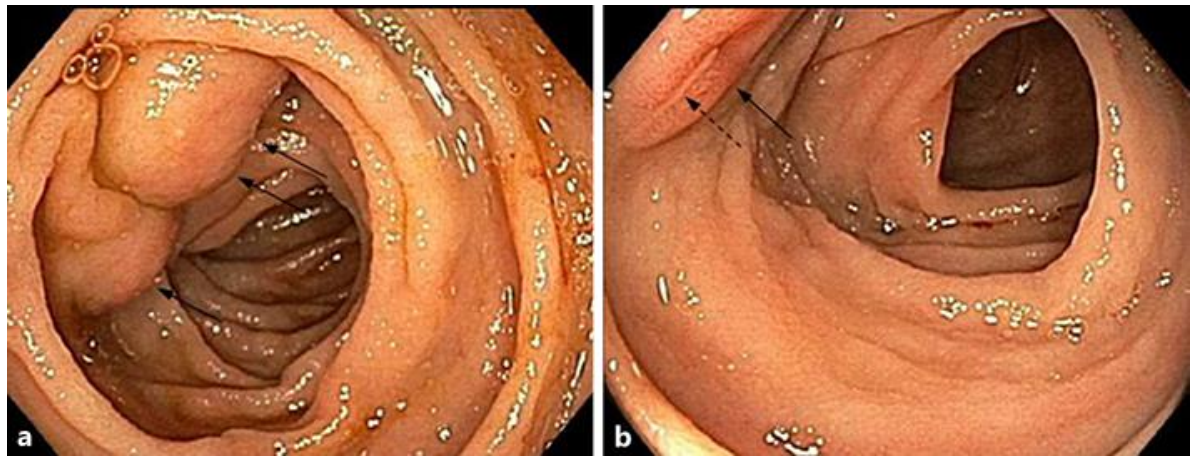

Fig. 3. a Endoscopic photograph of a duodenal varix involving the third portion of the duodenum (black arrows). $\mathbf{b}$ Endoscopic photograph of a duodenal varix involving the third portion of the duodenum (black solid arrow). Note the white spot (black dashed arrow) within an area of superficial erythema representing high-risk stigmata of bleeding. 